\title{
$T$ waves in Western Mediterranean Sea after the May 21, 2003 Algerian earthquake
}

\author{
Stefano Solarino $\left({ }^{1}\right)$ and Claudio Eva $\left(^{2}\right)$ \\ (1) Istituto Nazionale di Geofisica e Vulcanologia, Sezione CNT, Roma, Italy \\ (2) Dipartimento per lo Studio del Territorio e delle sue Risorse (DipTeRis), \\ Università degli Studi di Genova, Italy
}

\begin{abstract}
Aim of this paper is to discuss on the $T$ phases generated after the $m_{b} 6.5$ earthquake that shook Algeria on May 21 , 2003. The seismograms, recorded by a cluster of seismic stations located on the coast facing the Ligurian Sea, Northern Italy, some $800 \mathrm{~km}$ N-NE from the source, represent a good database able to shed some light on the recognition, propagation and characteristics of these quite uncommon phases. The occurrence and the recording of $T$ phases are in fact due to particular conditions, and require both particular characteristics of the bathymetric slope and the existence of a clear path between the instruments and the earthquake's source: these constraints are exactly realized in the north-western part of the Mediterranean Sea, whose coasts have been affected several times in the past by similar events. The preliminary investigations on the complex recorded seismogram show two different behaviours for stations close the coast and inland. In both cases, two distinct $T$ phases (namely $T 1$ and $T 2$ ) are observed. In one case they have apparent velocities close to an average SOFAR channel, and are thus the recording of direct $T$ phases. In particular, $T 1$ is probably a precursor due to some scattering, while $T 2$ is the direct $T$ wave. Conversely, the recordings of the stations inland show apparent velocities that suggest back conversion of the original $T$ to $P$ and $S$ waves and a crustal path. The frequency content of the $T$ phases, as derived from the spectral analysis, reveals marked amplitude peaks also in the range 1-3 Hz, conversely to what was proposed by other authors for similar occurrences in other parts of the world. Since the geometry and shape of the SOFAR channel vary, it is highly likely that the spectrum is biased by the water conditions and the frequency content might change in different seas. Finally, the attenuation of the $T$ phase does not depend on the actual distance of the receiver from the source but rather from the backconversion point: the amplitude varies thus with the in-land path and decreases proportionally to $x^{-1}$.
\end{abstract}

Key words T waves - Ligurian Sea - SOFAR - frequency content

\section{Introduction}

On May 21, 2003 Algeria was shaken by a strong $\left(m_{b}=6.5\right)$ earthquake the consequences of

Mailing address: Dr. Stefano Solarino, Istituto Nazionale di Geofisica e Vulcanologia, Sezione CNT, c/o Dipartimento per lo Studio del Territorio e delle sue Risorse (DipTeRis), Università degli Studi di Genova, V.le Benedetto XV 5, Genova, Italy; e-mail: peter@dipteris.unige.it which were some 2266 people killed, more than 10000 injured, 43500 buildings damaged or destroyed. The earthquake also caused a small tsunami in the Northern Mediterranean Sea recorded on tide gauges at Palma de Mallorca (Spain, $1.2 \mathrm{~m}$ ), Nice (France, $10 \mathrm{~cm}$ ), Genoa (Italy, $8 \mathrm{~cm}$ ). The earthquake was clearly felt along the coast of Liguria (north-western Italy), located some $800-900 \mathrm{~km}$ apart from the source, with an intensity of III-IV MSK scale, and it was recorded by the seismic stations operating in the Liguria Region (table I; figs. 1 and 2). The source of this shock was located close to the Algerian coast, with the following coordinates (from EMSC: http://www.emsccsem.org/): date - 21/05/03; origin time - 
Table I. Location (distance and azimuth) and instrumentation of the seismic stations used in this work. The distance from the coast computed on the incoming path is also reported.

\begin{tabular}{ccccc}
\hline \hline Station code & $\begin{array}{c}\text { Distance from } \\
\text { epicenter }(\mathrm{km})\end{array}$ & Azimuth $\left(^{\circ}\right)$ & $\begin{array}{c}\text { Distance from } \\
\text { the coast }(\mathrm{km})\end{array}$ & Instrument make and type \\
\hline OFIN & 887 & 25 & 45 & Geotech S13 (1c) \\
NEGI & 833 & 24 & 7 & Guralp CMG 40 (3c) \\
OIMI & 846 & 25 & 11 & Geotech S13 (1c) \\
MONE & 858 & 24 & 35 & Guralp CMG 40 (3c) \\
OPCP & 932 & 26 & 80 & Geotech S13 (1c) \\
GENL & 935 & 28 & 1 & Guralp CMG 40 (3c) \\
ROTM & 956 & 24 & 132 & Lennartz 3D 5s (3c) \\
OROB & 884 & 24 & 60 & Geotech S13 (1c) \\
RONM & 834 & 23 & 12 & Lennartz 3D 5s (3c) \\
\hline
\end{tabular}

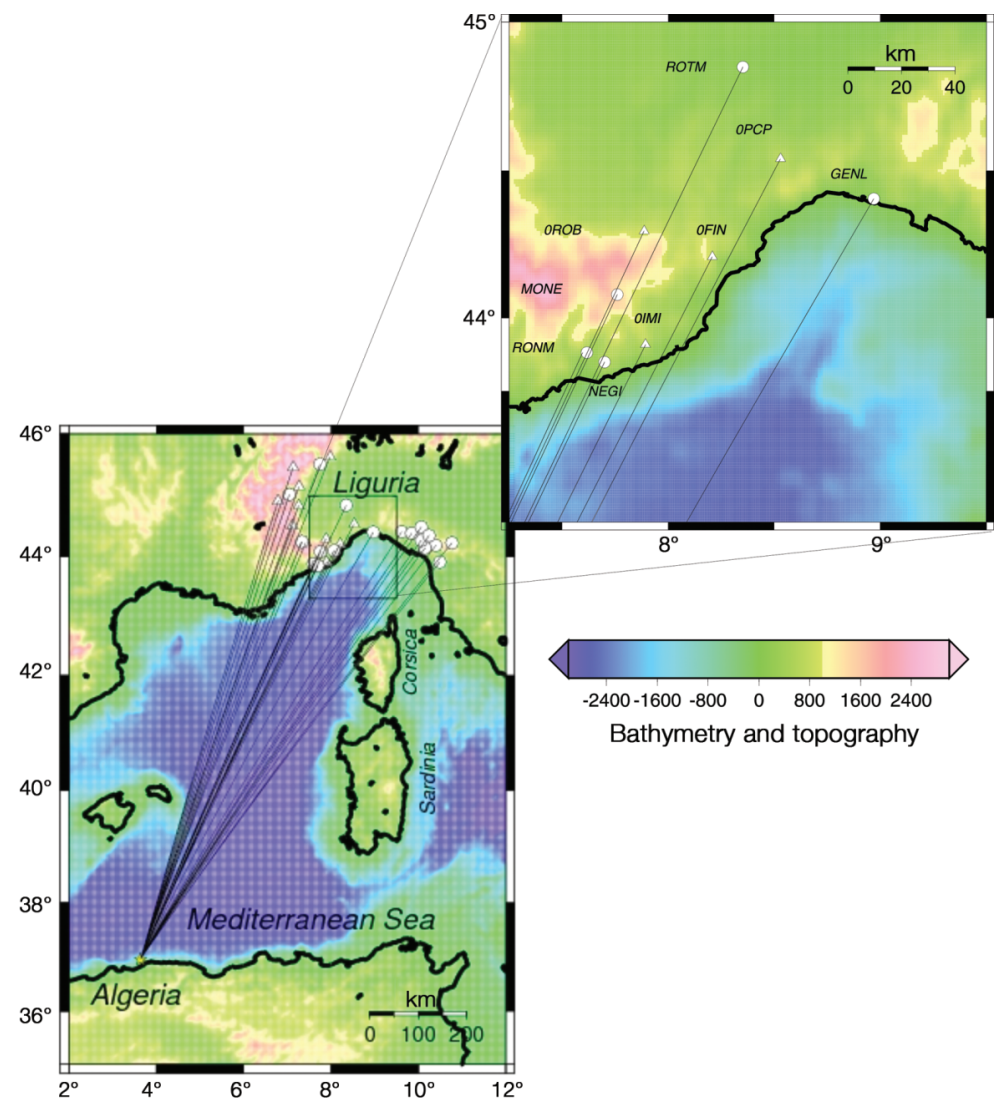

Fig. 1. Path connecting the epicenter (star) of the Algerian earthquake to RSNI recording stations in the Mediterranean Sea. Circles indicate the three-component stations and triangles the mono-component ones. The panel on the right hand-side shows a zoom on the stations used in this work. 


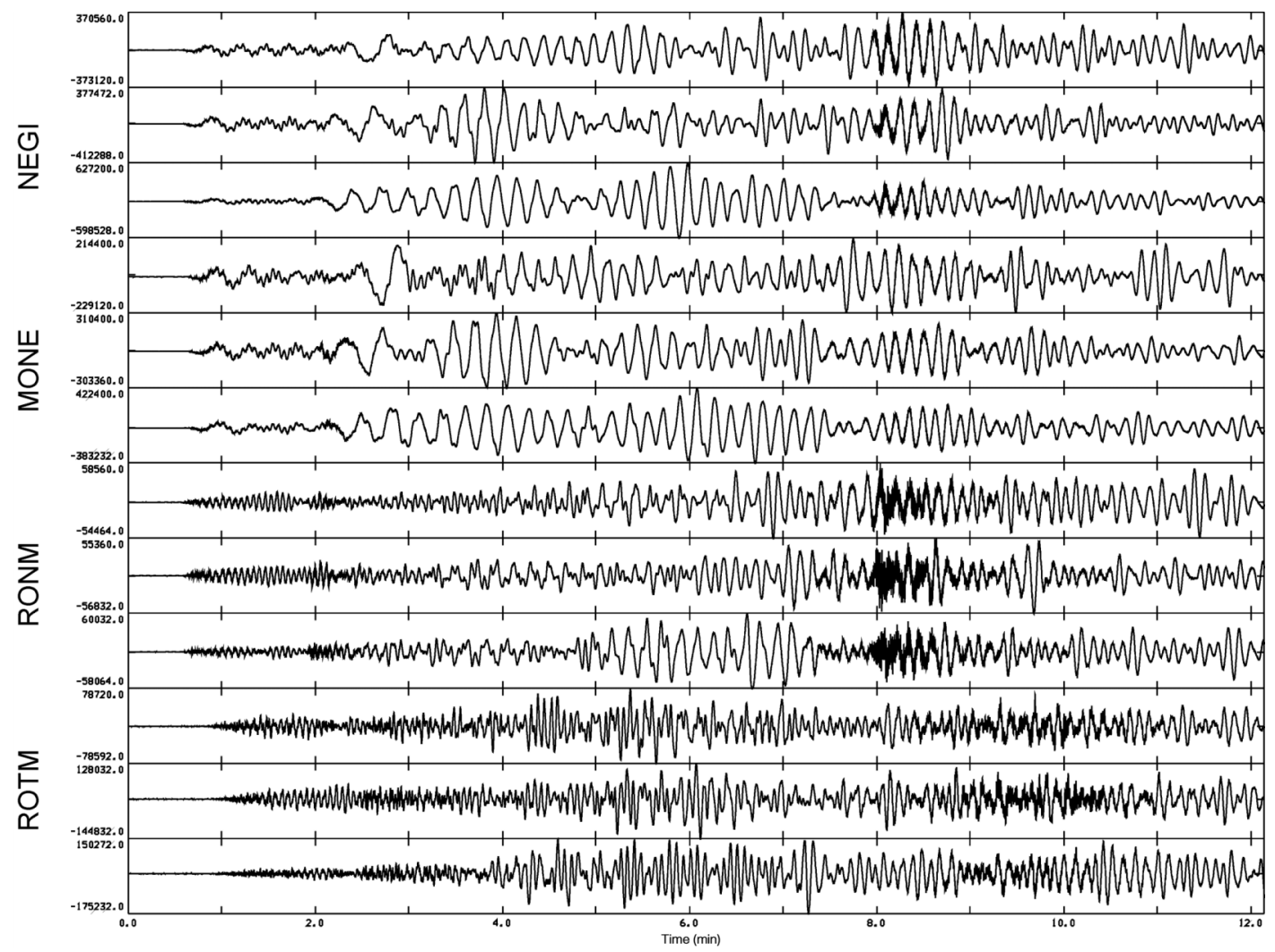

Fig. 2. Sample of the recordings of the Algerian earthquake as detected by some instruments of the RSNI network. NEGI and MONE are equipped with broadband instruments.

18:44:20; lat - 36.96N; long - 3.634E; depth $12 \mathrm{~km} ; m_{b}-6.5$.

Although no macroseismic survey has been carried out, the available reports on human perception of the event indicate that while the earthquake was clearly felt along the coastal line in Genova and Imperia (GENL and OIMI in fig. 1), where it created a certain alarm, the shaking was very weak or completely absent in land. Figure 3 shows the deconvolved estimate of the acceleration level (in $\mathrm{mm} / \mathrm{s}^{2}$ ) for stations NEGI, MONE and GENL. It is evident that MONE, that is $35 \mathrm{~km}$ away from the coast (fig. 1 ), experienced a significantly smaller acceleration, almost 6 times less, than GENL.

The recordings of the Algerian earthquake as detected by the velocity seismometers installed in north-western Italy show, approximately 8 to 10 min after the $P$ wave arrival, a strong and long perturbation which we attribute to $T$ phase (fig. 2). The amplitude of such a phase, at least two orders higher than the $P$ and $S$ waves in some recordings, and its frequency content were the reasons for confusing its occurrence with a local seaquake. However, the oceanic structure and the properties of water in the Western Mediterranean Sea, along with a rather developed SOFAR channel, favour the generation and propagation of $T$ waves (Jensen et al., 1994). In fact the geometry and shape of both Algerian and Western Ligurian coasts are able to convert a fraction of energy of seismic waves into acoustic energy when they hit the ocean boundary and to convert back this energy to seismic when the $T$ waves impact again on the 


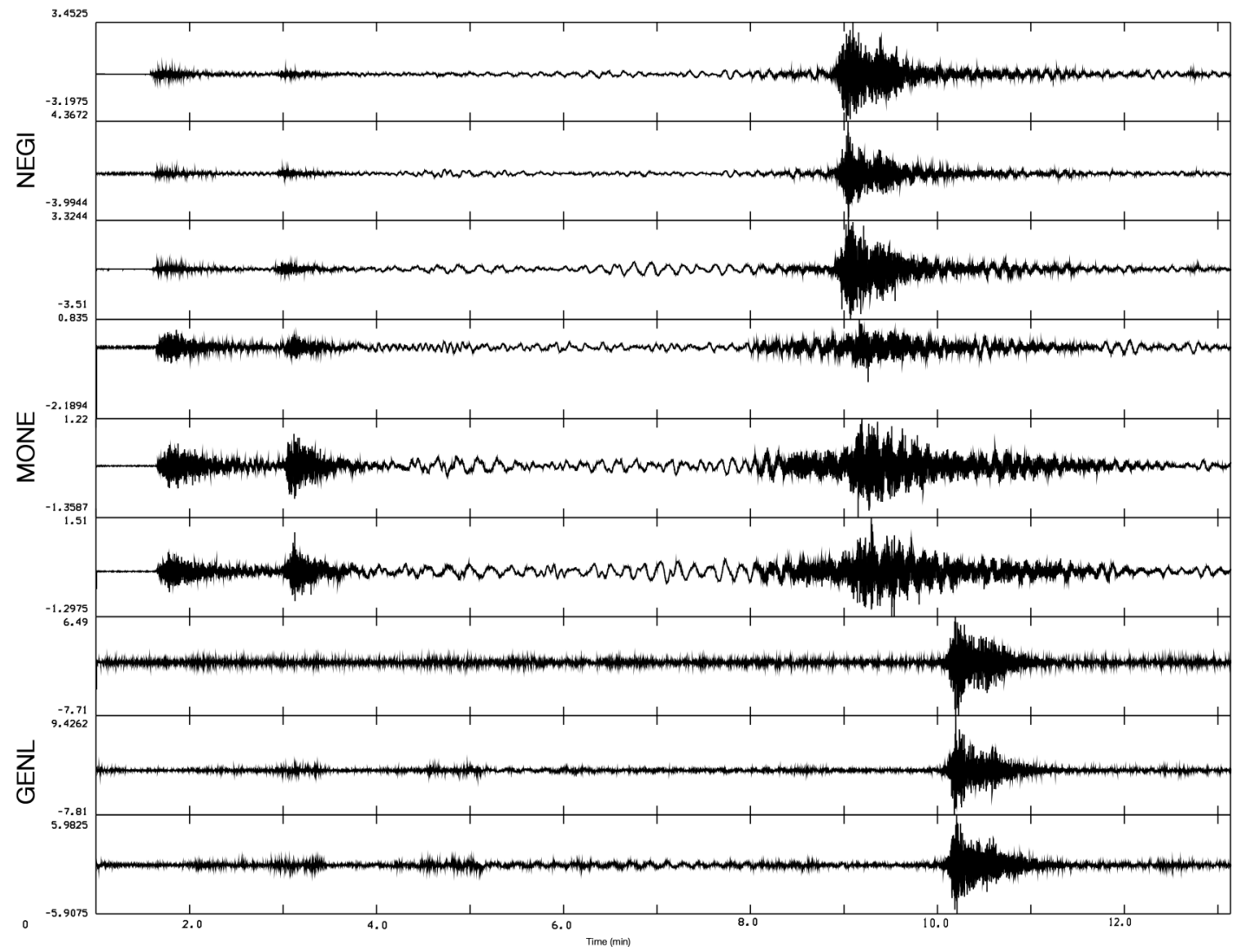

Fig. 3. Acceleration experienced by three sites NEGI, MONE and GENL (in $\mathrm{mm} / \mathrm{s}^{2}$ ).

slope (Talandier and Okal, 1979). The presence of two major islands, Corsica and Sardinia, prevent the Eastern Italian coast (Tyrrhenian side) being affected by such waves. Hence Western Liguria presents the most favourable conditions to observe $T$ waves propagating in the Western Mediterranean Sea when generated by Algerian quakes, and even if many examples of $T$ waves have been observed in the study area, the quake of May, 21st is one of the most felt by the population in the last fifteen years.

In the two weeks following the main shock some hundred aftershocks were recorded, contributing to a database of events with magnitude $\left(m_{b}\right)$ ranging from 3.7 to 6.5 . Although in most recordings the late phase that we hereby attrib- ute to $T$ phase is clearly visible, in this paper we only focus on the main shock to deploy our motivation for the attribution to such a phase and to investigate its spectral characteristics and inland propagation.

\section{Identification of $T$ phases}

The seismograms were analyzed taking into account recordings of the RSNI (Regional Seismic Network of north-western Italy), composed of 30 stations centralized in Genova, equipped with short period, enlarged band or broad band velocimeters. Among the available recordings, the stations along the same azimuthal range and 


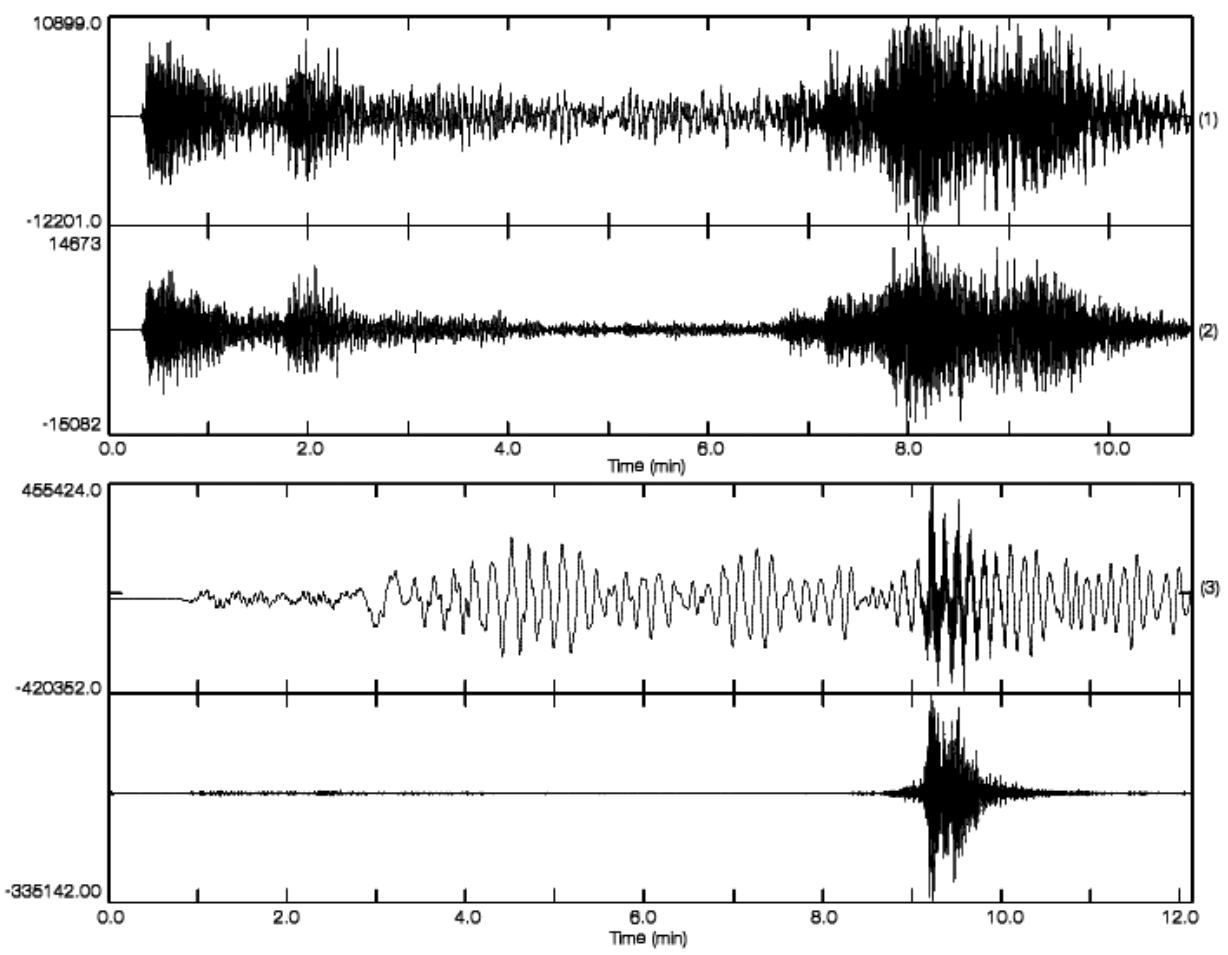

Fig. 4. Seismograms of the vertical components of ROB (upper) and GENL (lower) before and after applying the bandpass filter $0.5-20 \mathrm{~Hz}$.

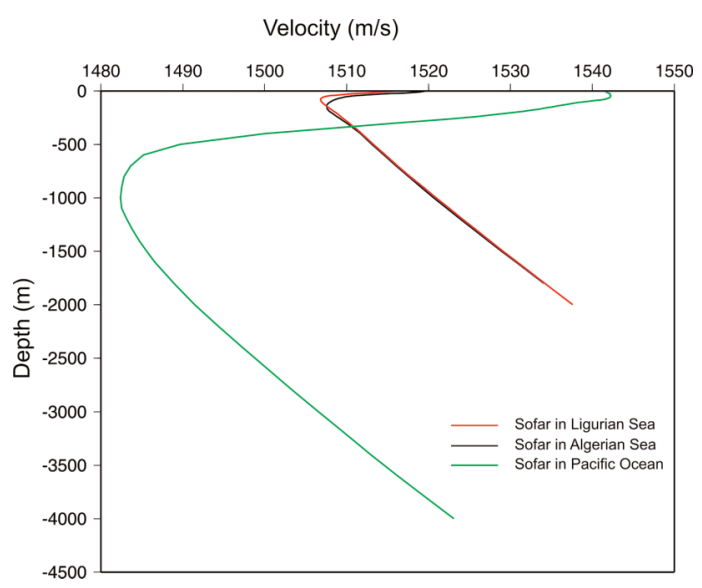

Fig. 5. Velocity of the SOFAR channel in May for the Mediterranean Sea. The SOFAR for the Pacific is reported for comparison. closer to the coast were selected (table I). Besides the instrumental characteristics, table I indicates distance and azimuth from the source for each station. The distance of each station from the coast, as computed on the incoming wavefront, is also reported; this measure is fundamental to distinguish and explain different seismic behaviours.

The available recordings of the Algerian earthquake are very different, being detected by various instruments (table I). Nevertheless, a high amplitude phase is clearly visible in the second part of each seismogram (figs. 2, 3, 4 and 6a,b), some 8 min after the $P$ and $S$ arrivals. Short period and semi-broad band instruments (ROTM and RONM in fig. 2) appear as the most sensitive, whereas this phase is partly masked by surface waves in broadband recordings (NEGI and MONE in fig. 2). It is evident that, before any further analysis is done, the 

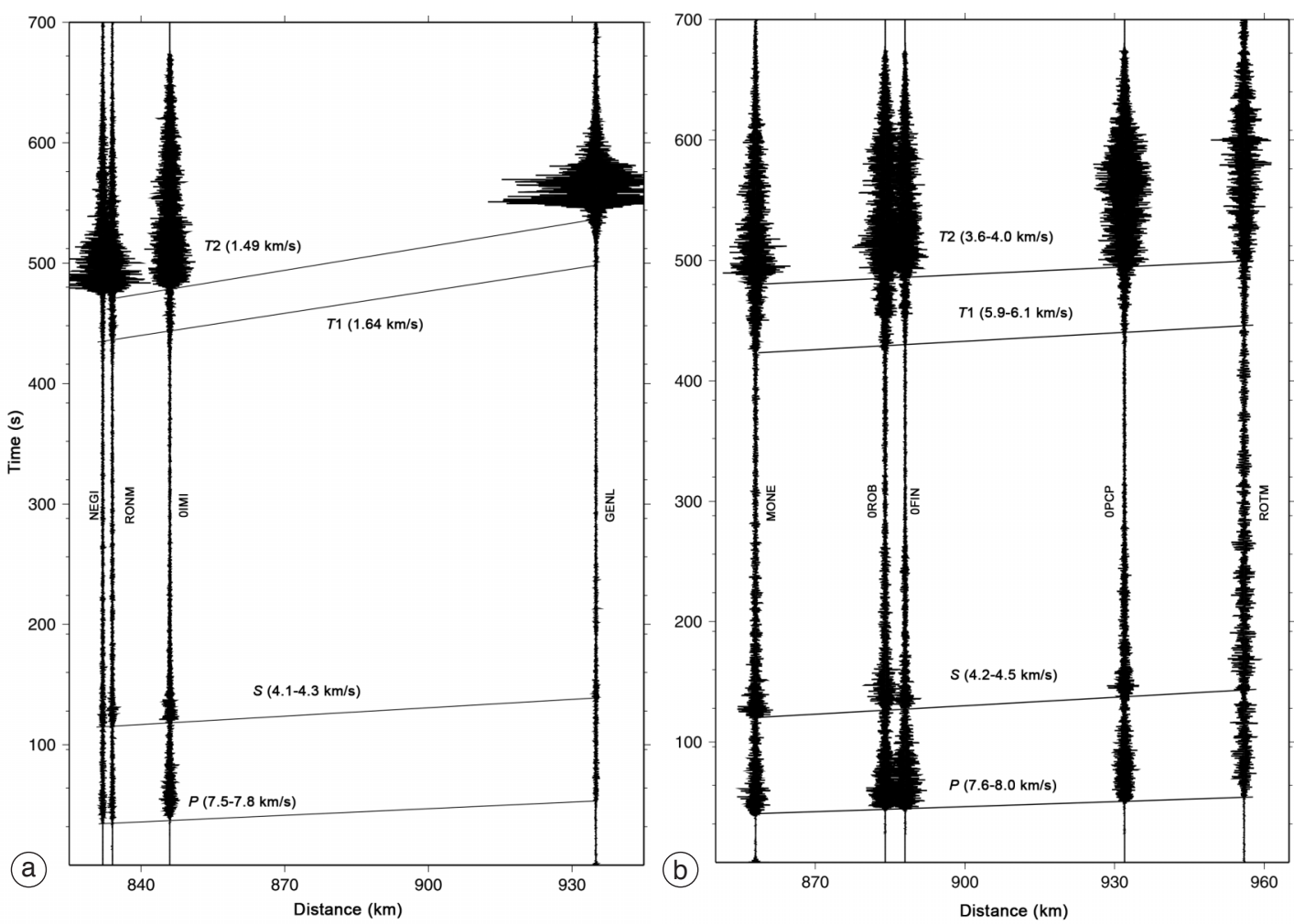

Fig. 6a,b. Seismograms of two groups (divided according to their location) of vertical components for stations ordered with respect to the epicentral distance: a) stations on the coast; b) stations in land. The recognized phases and their apparent velocities are also shown $(\mathrm{km} / \mathrm{s})$.

lower frequencies must be removed. According to the spectral analysis conducted on several seismograms, a $0.5-20 \mathrm{~Hz}$ band pass filter was applied to all records. More details on the spectral content of the wavelets will be given and discussed in the next paragraph. As an example, the appearance of the vertical component after filtering for GENL and ROB stations (which are broad band and short period respectively) is shown in fig. 4; no significant differences are found for the horizontal components.

Since the shape and characteristics of a $T$ phase strongly depend on the position of the recording station with respect to the coast, seismograms were separated into two groups, one relative to instruments operating close to the coast (fig. 6a), the other relative to stations located inland (fig. 6b). The first group is made of recording instruments located within a distance of $35 \mathrm{~km}$ from the coast computed on the incoming wavefront (see fig. 1); the second comprises the stations located at $35 \mathrm{~km}$ and more from the coast. The threshold to distinguish between groups was chosen by both a visual inspection of the waveforms and by checking match and homogeneity of several seismogram juxtapositions.

In all plots, two late phases, recorded $500 \mathrm{~s}$ after the $P$ onset, are visible. We call them $T 1$ and $T 2$ respectively. The absolute path time, the shape and the amplitude of these phases are consistent with a $T$ wave or a back-converted $T$ wave. In fact, only by travelling with a velocity close to $1.5 \mathrm{~km} / \mathrm{s}$ can a wave reach a station located some 800 to $1000 \mathrm{~km}$ away from the source $8 \mathrm{~min}$ after the origin time. Moreover, the sonograms computed for the whole seismograms (some are shown in fig. 8) 
do not reveal any dispersive behaviour of the seismic waves, confirming that only body waves are contained within. All these are very sound elements to label the later arrival as $T$ phases.

The plots in fig. 6a,b allow us to compute the apparent velocities of the two groups of recordings. It is very clear that all stations close to the coast (fig. 6a) have recorded phases with an apparent velocity ranging from 1.5 to $1.65 \mathrm{~km} / \mathrm{s}$, another element that favours the recognition of $T$ phases.

A very different situation is observed for the recordings inland (fig. 6b). The apparent velocities are coherent with those of $P$ and $S$ waves travelling in the upper crust. These recordings are then relative to converted $T$ phases.

To summarize, the $T$ phase travelled in the SOFAR and reached the coastal station right after the conversion, conserving the velocity and energy of the $T$ wave. The conversion back to $P$ and $S$ must have happened very close to the coast. In fact, unlike what happens in other seas, the SOFAR low velocity waveguide in the Mediterranean is very shallow and narrow (fig. 5). The impact of the wave train with the slope must therefore have occurred at a distance from the coast where the bathymetry is compatible with the depth of the SOFAR waveguide, that is about $200 \mathrm{~m}$. By looking at the bathymetry, the conversion point should be at less than $6-8 \mathrm{~km}$ in average from the coast.

\section{Spectral characteristics and attenuation of the $T$ waves}

The results of the analysis in the frequency domain for the $T$ phase (namely for the part of the signal ranging from 7 to $10 \mathrm{~min}$ ) has been compacted in the plot shown in fig. 7. It reports the spectra of the non filtered vertical component of 5 recording stations (GENL, NEGI, RONM, MONE and ROTM) and reveals the existence of two frequency bands: the first in the range 0.01$0.5 \mathrm{~Hz}$ (which is not taken into account as excluded by the filtering applied in all analyses in the time domain) and the second in the range 0.5-20 Hz. Moreover the spectra exhibit peaks with maximum of amplitude in the range 1 to $3 \mathrm{~Hz}$, in agreement with the results obtained by other authors studying similar waves for the Pacific ocean paths (Koyonagi et al., 1995; Okal and Talandier,

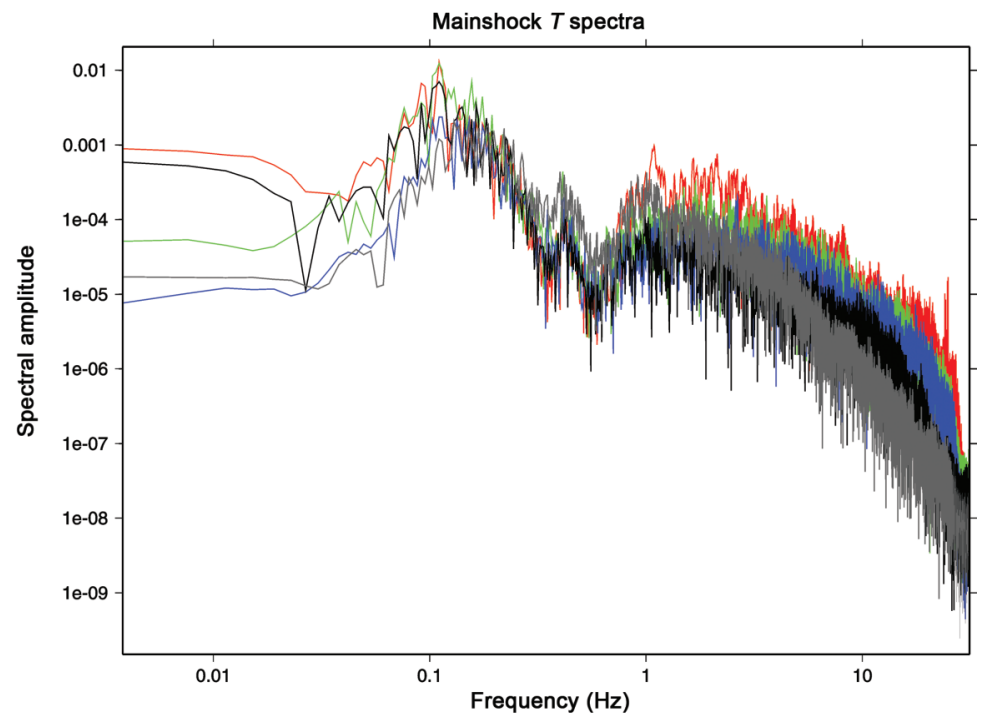

Fig. 7. Spectra of the presumed $T$ phase for stations GENL (red line), NEGI (green line), RONM (blue line), MONE (black line) and ROTM (grey line). 
1997; Okal, 2001). However some of these authors state that the spectrum of $T$ waves, as guided waves through the narrow SOFAR channel, is restricted to high frequencies, $f>2 \mathrm{~Hz}$ while in this case even lower frequencies are present. This difference can be related to the different properties of the SOFAR channel in the different seas, as we will point out in more detail later on. Spectra show a small shift of maximum amplitude towards higher frequencies with increasing coastal

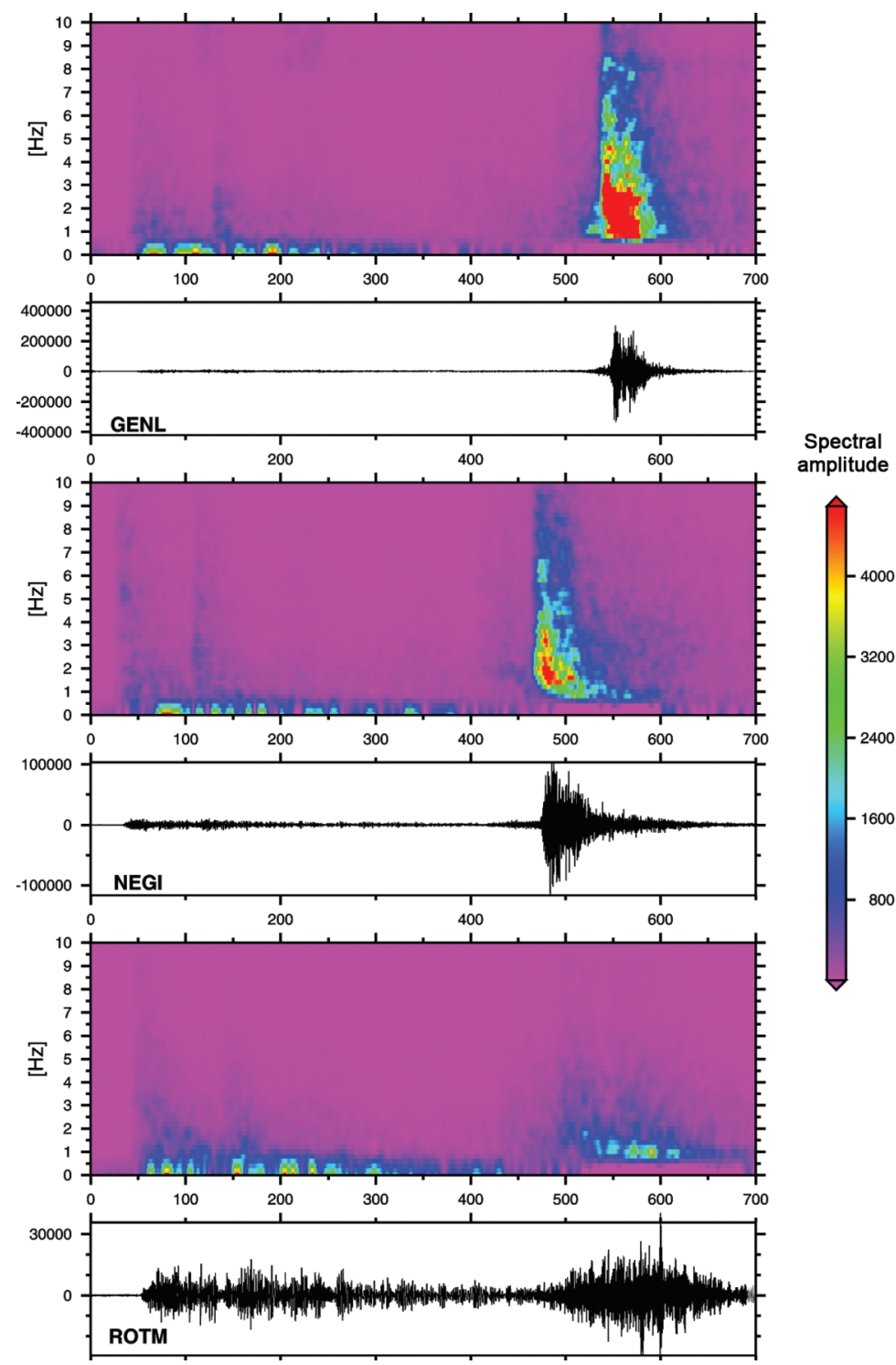

Fig. 8. Sonograms for GENL, NEGI and ROTM. 


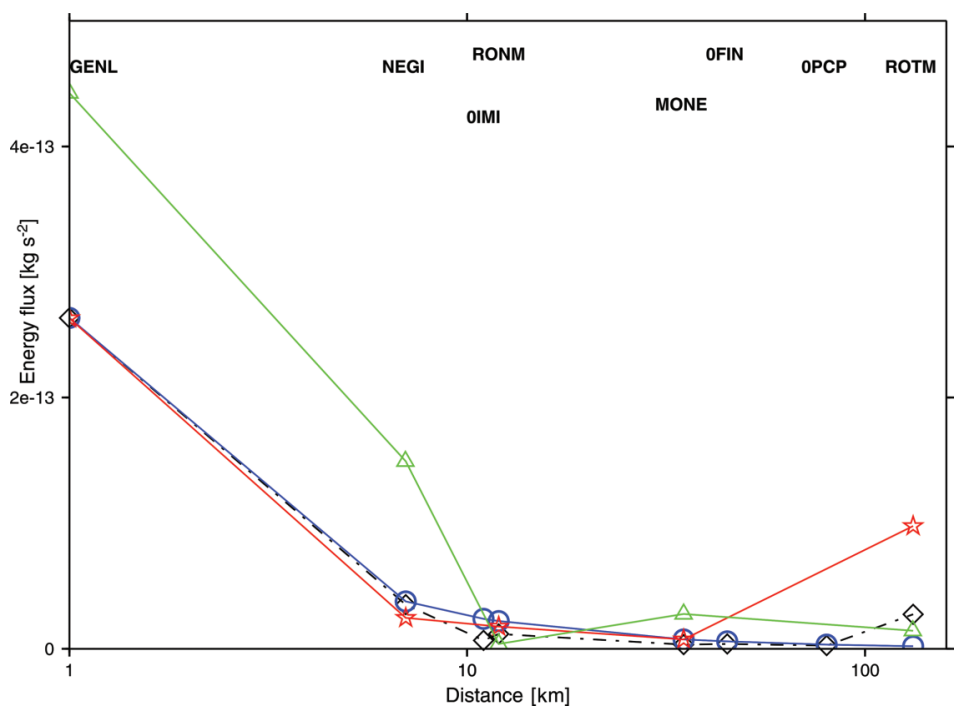

Fig. 9. Energy attenuation for each component with increasing distance computed in the frequency window 2$10 \mathrm{~Hz}$. Each symbol represents the energy computed at the relevant station. To fit all curves in one plot, the two transversal components (red and green line) are multiplied by 1e-6; no exaggeration has been applied to the vertical component (dotted line). The shape of the curve attenuating with $R^{-1}$ is reported for comparison (blue line).

distance (from red to grey lines), but substantially the higher values are mainly centred in the band 1-2 Hz. Finally, as previously stated for the whole spectrum, longitudinal and vertical components show a similar trend.

Coming to a more quantitative aspect, a preliminary approach to the attenuation of $T$ waves has been performed. For this purpose, the $T$ phase energy flux, that is the integral of the $T$ phase spectrum in the frequency range $2-10 \mathrm{~Hz}$, was evaluated. Following Newman and Okal (1998) and Okal et al. (2003)

$$
E=\frac{(\alpha \rho)}{\pi} \int_{\omega \min }^{\omega \max } \omega^{2}\left|U\left(\omega^{2}\right)\right| \mathrm{d} \omega
$$

where $U$ is the complex spectral amplitude of the ground motion and $\rho$ and $\alpha$ are the density and the $P$ wave velocity of the receiver structure respectively.

The results, as a function of distance from the coast on the travel path, are presented in fig. 9, showing how the energy of converted $T$ waves attenuates with the inland path.
Comparing the actual attenuation of $T$ waves with geometrical spreading (proportional to $x^{-1}$ ) it is possible to appreciate the good agreement between the two curves. Again, no significant differences have been inferred for horizontal components.

A visible anomaly can be observed for station ROTM: indeed this station shows a very different spectrum (fig. 7, grey line) and an anomalous increase in amplitude (fig. 9, right handside). This station is located on the very thick sedimentary deposits of the Po Plain domain: it is plausible to ascribe this anomalous behaviour to a local site effect. Finally it is interesting to notice that the energy decreases with distance on the incoming path from the coastal line rather than the source distance (table I). For example, MONE is one of the closest stations $(858 \mathrm{~km}$ from the source), but it is at $35 \mathrm{~km}$ from the coast, that is about $41-43 \mathrm{~km}$ from the likely conversion point. On the other hand, GENL is one of the more distant stations $(935 \mathrm{~km}$ from the proposed source) but it is only a few km away from the conversion point. The perception of the 


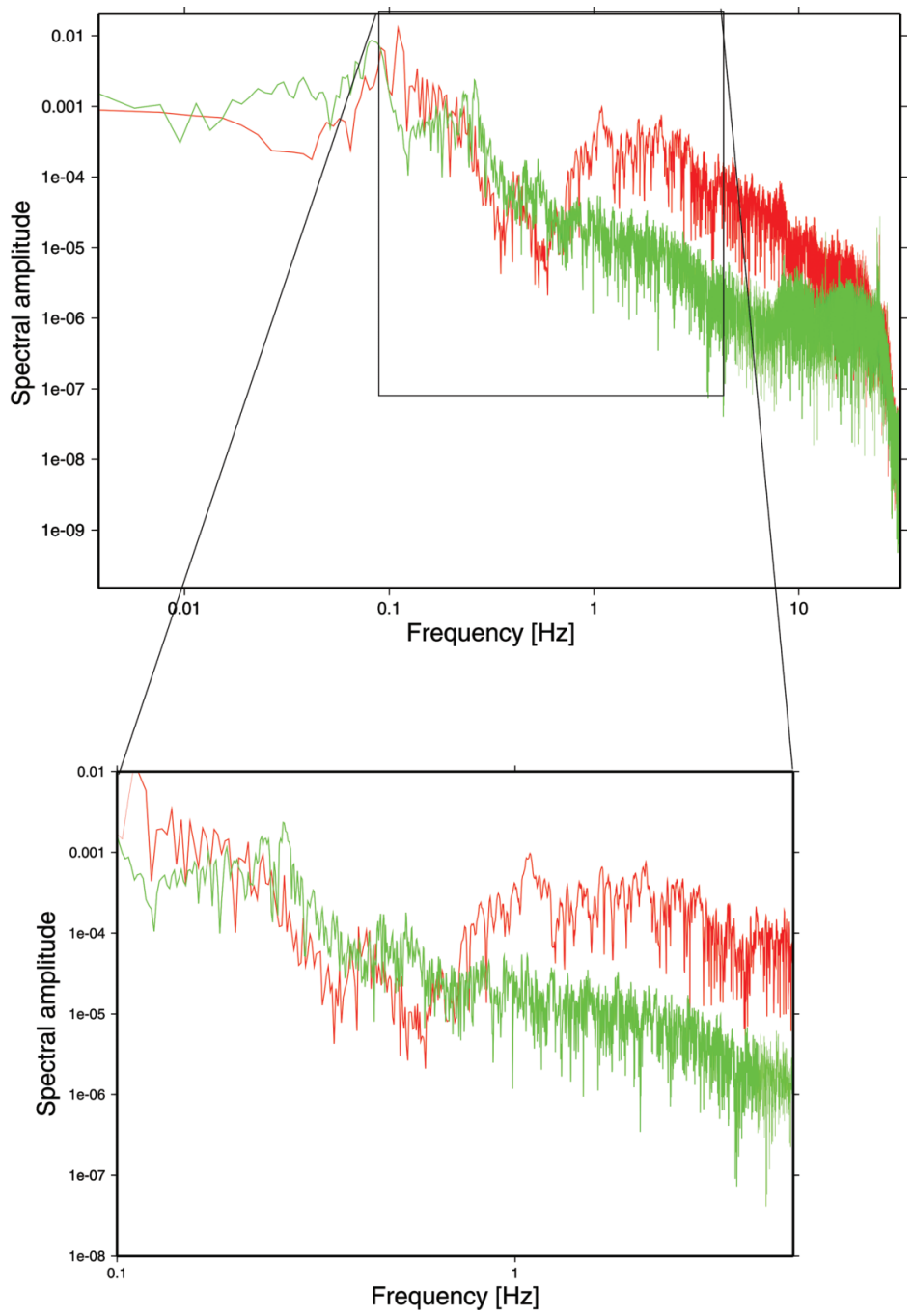

Fig. 10. Comparison of $T$ wave spectrum (red line) and $P S$ spectrum (green line) for GENL station.

earthquake, which was reported to be so different for towns that are very close, is certainly related to the distance from the conversion point. In fact, as already pointed out, most of the reports were collected in Genova and Imperia, located at 1 and $11 \mathrm{~km}$ respectively from the coast. Finally, it must be remarked that an energy loss of about $80 \%$ is already reached when the path exceeds $10 \mathrm{~km}$.

\section{Discussion and conclusions}

This paper analyzed the characteristics of late seismic phases associated with the $m_{b}=6.5$ earthquake that shook Algeria on May 21, 2003 as recorded by seismic stations installed on the coast facing the Ligurian Sea.

These stations, located along the north north-east path at a distance ranging from 800 
to $1000 \mathrm{~km}$, provided seismograms in which, at about 7-8 min after the $P$ phase, a complex $T$ wave is clearly visible. The identification of this later phase as $T$ has been proposed on the basis of several pieces of evidence. The shape, amplitude and path time are consistent with those met in other studies and with a wave travelling with a velocity of $1.5 \mathrm{~km} / \mathrm{s}$. The apparent velocity computed for the recordings on stations close to the coast is of the same order. Sonograms make the exclusion of surface waves within the seismogram very reasonable.

On all recordings, the $T$ train is split into two parts. In particular, the stations that are apart from the coast show apparent velocities compatible with $P$ and $S$ waves that travelled within the crust. They are therefore the recording of back-converted $T$ waves. Instead, the recordings of the stations closer to the coast, and therefore to the conversion point, exhibit original $T$ waves.

The preliminary study on the spectral characteristics and attenuation of the recorded $T$ waves provided interesting insights on the propagation of such a wave in the Mediterranean Sea. They are:

- The frequency content extends down to 1 $\mathrm{Hz}$, conversely to what was proposed by other studies (Okal and Talandier, 1997; Talandier and Okal, 1998). Moreover, sonograms and spectra show that the maximum amplitude is confined in the range $1-3 \mathrm{~Hz}$, while in other studies values around $5 \mathrm{~Hz}$ have been suggested. We propose that the shape and depth of the SOFAR waveguide channel may influence the spectra, since in fact the characteristics of the SOFAR in the Mediterranean Sea are very different from other seas.

- The likely position of the back-conversion point is very close to the coast, where the bathymetry is of the same order of the depth of the waveguide (around $200 \mathrm{~m}$ ). Because of the shape of the slope under the Ligurian Sea, that is incised by several steep canyons, it is highly likely that the conversion point is at less than 10 $\mathrm{km}$ from the coast.
- $T$ waves attenuate very fast in the vicinity of the conversion point. We estimate that $80 \%$ of the energy is dumped at a distance greater than 10 to $15 \mathrm{~km}$ beyond the conversion point.

- Compared to $P$ and $S$ waves, $T$ phases contain more high frequency and generally speaking, of higher amplitude (fig. 10).

\section{Acknowledgements}

The paper profitted from the comments and suggestions of two anonymous reviewers.

The experience of Giacomo Carenzo, who shared the thrill of one night spent doing phase picking, was of great help for the accomplishment of this work.

\section{REFERENCES}

Jensen, F.B., W.A. KuPERman, M.B. Porter and H. SchmidT (1994): Computational Ocean Acoustics (Am. Inst. Phys. Press, New York), pp. 612.

Koyanagi, S., K. AKI, N. Biswas and K. Mayeda (1995): Inferred attenuation from site effect-corrected $T$ phases recorded on the Island of Hawaii, Pure Appl. Geophys., 144, 1-17.

Newman, A.V. and E.A. OKal (1998): Teleseismic estimates of radiated seismic energy: the $E / M_{0}$ discriminant for tsunami earthquakes, J. Geophys. Res., 103, 26885-26898.

OKAL, E. (2001): Converted $T$ phases recorded on Hawaii from Polynesian nuclear tests: a preliminary report, Pure Appl. Geophys., 158 (3), 457-474.

OKAL, E. and J. TALANDIER (1997): $T$ waves from the great Bolivian deep earthquake in relation to channeling of $S$ wave energy up to the slab, J. Geophys. Res., 102 (B12), 27412-27437.

Okal, E., P.J. Alasset, O. Hyvernaud and F. Schindele (2003): The deficient $T$ waves of tsunami earthquakes, Geophys. J. Int., 152, 416-432.

TALANDIER, J. and E. OKAL (1979): Human perception of $T$ waves: the June 22, 1977 Tonga earthquake felt on Tahiti, Bull. Seismol. Soc. Am., 69 (5), 1475-1486.

TALANDiER, J. and E. OKal (1998): On the mechanism of conversion of seismic waves to and from $T$ waves in the vicinity of island shores, Bull. Seismol. Soc. Am., 88 (2), 621-632.

(received January 20, 2006; accepted November 7, 2007) 